\title{
Dipterologische Beiträge.
}

Von V. v. Röder in Hoym (Herzogthum Anhalt).

I.

In meinem Garten $z u$ Hoym sammle ich alljährlich Tachina lepida M. in beiden Geschlechtern. Meigen stellt diese Art zur Gattung Leucostoma, Schiner zur Gattung Phyto. Ich finde aber, dass sie zu keiner von beiden Gattungen gehört, sondern zu Rhinophora. Sie besitzt auf den Wangen unten neben dem Augenrande eine kurze Reihe von Börstchen, wie dieses bei den Rhinophora-Arten der Fall ist. Mithin muss die Art Rhinophora lepida M. heissen. Die Börstchen auf den Wangen sind bei ihr zwar sehr fein; eben wegen dieser Börstchen lässt sie sich nur zur Gattung Rhinophora bringen; sie bildet aber einen unverkennbaren Uebergang zur Gattung Leucostoma.

Das Weibchen der in Rede stehenden Art ist noch nicht beschrieben. Die Beschreibung möge hier folgen.

\& Augen weit auseinander stehend, durch eine schwarze Mittelstrieme getrennt, diese ist auf beiden Seiten von den weissschimmernden Orbiten eingefasst. Hinterleib glänzend schwarz, ohne weisse Binden. Flügel etwas bräunlich tingirt.

Ich erhielt diese Art auch vom Herrn Gymnasiallehrer P. Stein in Genthin (Preussen), von Herrn van der Wulp aus Holland und Herr von Bergenstaum schrieb mir, dass er sie auf dem Schneeberg bei Wien gefangen habe.

\section{II.}

An demselben Orte in meinem Garten, wo ich Rhinophora lepida M. fand, fing ich die seltene Frauenfeldia rubricosa M. in beiden Geschlechtern.

\section{III.}

Z etterstedt beschreibt in den Dipt. Scand. (Bd. III, p. 1091) Tachina Pavoniae. Diese Art wurde aus Pavonia carpini gezogen. Es ist dieselbe Art, welche $\mathrm{Z}$ etterstedt als Tachina grandis beschrieb und die Rob. Desvoidy (Histoire naturelle des Dipt. des environs de Paris, Bd. I, p. 256) Scotia Saturniae R. D. nennt. Es fehlen ihr die Macrochaeten auf der Mitte der mittleren Hinterleibsringe. Sie gehört zur Gattung Exorista.

\section{Exorista (Tachina) pavoniae Zett.}

Syn. Tachina grandis Zett. und Scotia saturniae Rob. Desv. 
IV.

In der Wiener Entomolog. Zeitung, 1887, pag. 155, habe ich die Gnoriste-Arten aufgezählt. Damals kannte ich Gnoriste trilineata Zett. (Dipt. Scand. XI, 4095, 3) nicht. Ich sammelte diese Art in beiden Geschlechtern im Harz, an der Strasse von Mägdesprung nach Harzgerode, Anfangs Juni.

Gnoriste trilineata Zett. ist das Männcben zu Gnoriste bilineata Zett. (Dipt. Scand. XI, p. 4094) @. Es ist daher die Art als Gnoriste trilineata Zett. *) zu bezeichnen. Sie ist bisher nur in Norwegen gefunden worden.

Von den exotischen Gnoriste-Arten habe ich eine übersehen mit aufzuzählen; es ist Gnoriste chilensis Philippi.

Bisher sind folgende Arten bekannt geworden:

Gnoriste apicalis M., Mecklenburg, Dorpat, Berlin.

Gnoriste trilineata Zett. ơ, Norwegen, Harz.

Syn. Gnoriste bilineata Zett. $Q$ und Gnoriste bivittata Schumm. $q$.

Gnoriste longirostris Siebke, Norwegen.

Gnoriste Harcyniae von Röder, Harz.

Gnoriste megarrhina O. S., Nordamerika.

Gnoriste chilensis Phil., Chili.

\section{V.}

Herr Realgymnasiallehrer E. Girschner in Meiningen sammelte auf dem Stedtlinger Moor in Thüringen eine sehr interessante Art der Gattung Hybos M., welche von Zetterstedt als Ocydromia nigripes Zett. (Dipt. Scand., Bd. I, p. 240) beschrieben wurde. Schiner führt diese Art als ihm nicht bekannt an. Z etterstedt sagt, dass dieselbe mit Hybos verwandt sei, aber sich durch die Hinterschenkel unterscheidet. Doch gehört diese Art sicher zu Hybos und nicht zu Ocydromia, wie uns das Flügelgeäder belehrt. Sie wird also in Zukunft Hybos nigripes Zett. beissen müssen.

\section{VI.}

Auf dem oben erwähntẹ Stedtlinger Moor in Thüringen fing Herr E. Girschner Dolichopus Stenhammari Zett., eine bisher nur aus Schweden und Lappland bekannt gewordene Art; für Deutschland ist sie neu.

*) Auch Gnoriste bivittata Schummel gehört zu Gnoriste trilineata Zett., wie die Beschreibung beweist. 


\section{$2 \mathrm{BHL}$ Biodiversity Heritage Library}

Röder, Victor von. 1888. "Dipterologische Beiträge." Wiener entomologische Zeitung 7, 95-96. https://doi.org/10.5962/bhl.part.27347.

View This Item Online: https://www.biodiversitylibrary.org/item/43819

DOI: https://doi.org/10.5962/bhl.part.27347

Permalink: https://www.biodiversitylibrary.org/partpdf/27347

\section{Holding Institution}

Smithsonian Libraries

\section{Sponsored by}

Smithsonian

\section{Copyright \& Reuse}

Copyright Status: NOT_IN_COPYRIGHT

This document was created from content at the Biodiversity Heritage Library, the world's largest open access digital library for biodiversity literature and archives. Visit BHL at https://www.biodiversitylibrary.org. 